\title{
Diagnostic methods for silica-reinforced carbon nanotube-based nanocomposites
}

\author{
M. K. $\operatorname{Eseev}^{1}$, A. A. Goshev ${ }^{1}$, P. Horodek ${ }^{2,3}$, S. N. Kapustin ${ }^{1}$, A. G. Kobets ${ }^{2,4}$, C. S. Osokin ${ }^{1}$ \\ ${ }^{1}$ Northern Arctic Federal University, Arkhangelsk, Russia \\ ${ }^{2}$ Joint Institute for Nuclear Research, Dubna, Moscow region, Russia \\ ${ }^{3}$ Institute of Nuclear Physics Polish Academy of Sciences, Krakow, Poland \\ ${ }^{4}$ Institute of Electrophysics and Radiation Technologies NAS of Ukraine, Kharkov, Ukraine \\ m.eseev@narfu.ru
}

PACS 62.23.St; 73.63.-b; 61.48.De

DOI 10.17586/2220-8054-2016-7-1-180-184

This paper presents results of the experimental studies of the properties of silica-based nanocomposites with filler in the form of carbon nanotubes by dielectric relaxation and positron annihilation spectroscopy. Based on these results, techniques for diagnosis and control of the investigated materials were proposed.

Keywords: carbon nanotube, nanocomposites, dielectric properties, positron annihilation spectroscopy.

Received: 20 November 2015

\section{Introduction}

Active research of composite materials, which include nanostructures such as carbon nanotubes (CNTs) as filler, is being conducted globally. Due to the large surface area of CNT, as well as significant differences in the physical properties between nanoobjects and macroscopic material, the properties of nanocomposites are not additive characteristics of each phase and can be radically different from those of each component's [1]. The data on the study of the electrical and other properties of nanocomposites with CNT is provided in these reviews [2,3]. A particularly urgent task is to study the strongly nonlinear dependence of the composite properties on the filler concentration, which is necessary for the selection of the optimum functional properties for the materials (strength, thermal, dielectric and other characteristics). Data on the influence of impurities on the dielectric properties of CNTs of different composites at microwave frequencies, associated with the search for promising materials for "stealth"-technologies, have been presented in the literature [4]. This paper [5,6] investigates the microwave characteristics of nanocomposite materials via controlled modification of the CNTs. We were also interested how the positrons will annihilate in materials reinforced with CNTs.

\section{The electrical properties of nanocomposite}

Our composite is based on $\mathrm{SiO}_{2}$. It is reinforced with multi-walled CNTs at different concentrations $-0 \%, 0.05 \%, 0.1 \%, 0.5 \%$ (by weight of silica + water), with an aspect ratio of $10-10^{2}$ (see Fig. 1), obtained by CVD-method.

Silicon was obtained from silicate glue. The chemical reaction is shown below.

$$
\mathrm{Na}_{2} \mathrm{SiO}_{3}+2 \mathrm{HCl} \rightarrow 2 \mathrm{NaCl}+\mathrm{H}_{2} \mathrm{SiO}_{3}, \quad \mathrm{H}_{2} \mathrm{SiO}_{3} \rightarrow \mathrm{H}_{2} \mathrm{O} \uparrow+\mathrm{SiO}_{2} .
$$

Electrical characteristics of the samples were studied by dielectric relaxation spectroscopy, according to which, the sample is placed between the plates of the capacitor (area of $S=16 \mathrm{~cm}^{2}$ and the distance between the plates $d=3.3 \mathrm{~mm}$ ). The sample was then exposed to an alternating 


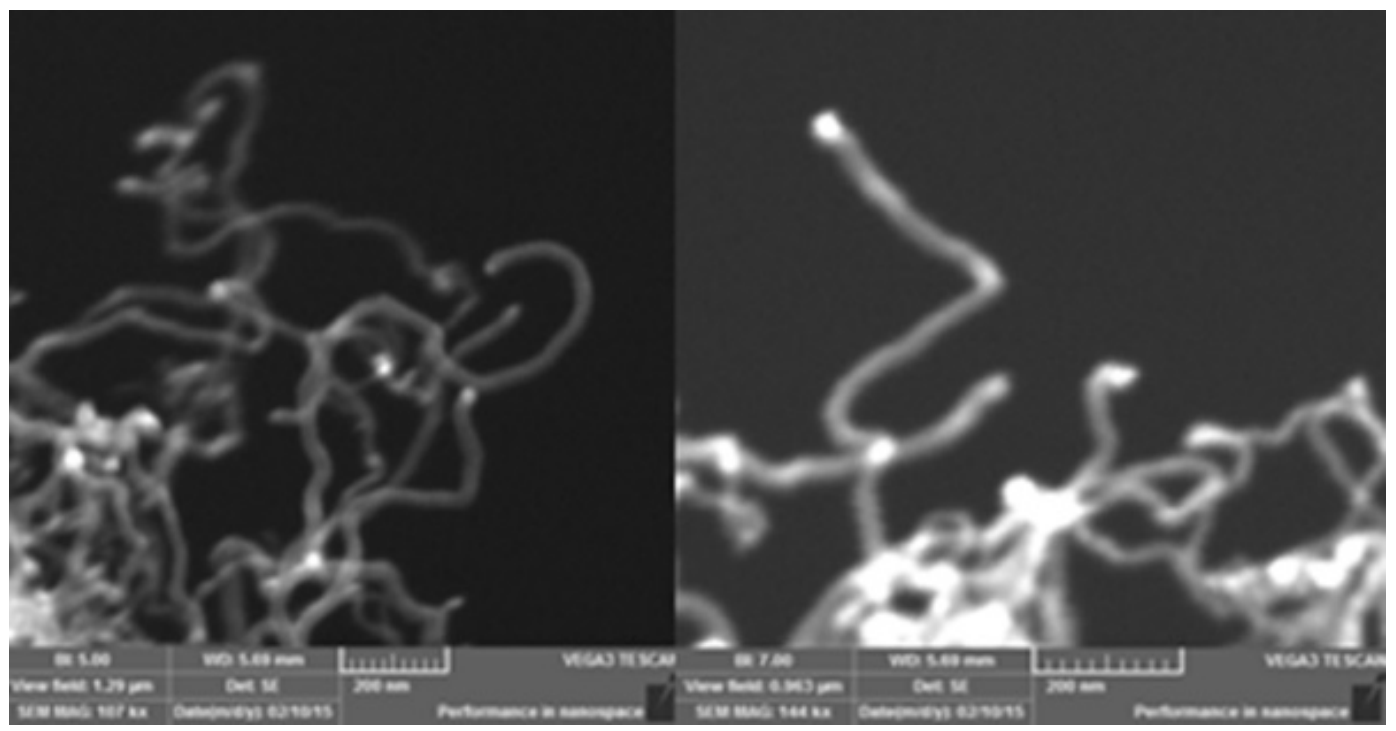

FIG. 1. SEM images of CNTs

electric field with a frequency varying from $25 \mathrm{~Hz}-1 \mathrm{MHz}$. The measured values were: electrical capacitance $C$, the quality factor $Q$, and the resistance $R$. The electrical properties of the composites in electric fields were measured using an immittance meter E7-20.

The microwave properties of the composite are described by the frequency of electrical field $f$ dependence of the complex permittivity and conductivity:

$$
\begin{gathered}
\varepsilon=\varepsilon^{\prime}-i \varepsilon^{\prime \prime}, \quad \varepsilon^{\prime}(f)=\frac{\left(C(f)-C_{0}\right) d}{\varepsilon_{0} S}, \quad \varepsilon^{\prime \prime}(f)=\frac{d}{\varepsilon_{0} S}\left(\frac{C(f)}{Q(f)}-\frac{C_{0}}{Q_{0}}\right), \\
\sigma(f)=2 \pi f \varepsilon^{\prime \prime}(f) \varepsilon_{0},
\end{gathered}
$$

where the real and imaginary parts characterize the dielectric polarization and losses in the composite respectively; $C, C_{0}$ - capacitance of the cell with the composite and empty cell respectively and $Q, Q_{0}$ - quality factor of the filled and empty cells.

According to the table, it can be argued, that there is an optimum concentration at which maximum absorption and scattering of electromagnetic waves at low frequencies occurs. It is known that a change in the CNTs concentration leads to a nonlinear modification of the nanomaterial's functional properties. This dependence is noted for the mechanical properties in [7]. The dependence of the silicon dioxide powder's electrical properties (1), (2) on the concentration of CNTs at different frequencies is shown in Table 1.

TABLE 1. The dependence of electrical properties upon different frequencies

\begin{tabular}{|c|c|c|c|c|c|c|}
\hline \multicolumn{2}{|c|}{ Composition, CNT, n \% } & REF, 0 & 0.01 & 0.05 & 0.1 & 0.5 \\
\hline \multirow{3}{*}{$f=50 \mathrm{~Hz}$} & $\varepsilon^{\prime}, 10^{4}$ & 59.4 & 53.6 & 326.3 & 41.9 & 8.8 \\
\cline { 2 - 7 } & $\varepsilon^{\prime \prime}, 10^{4}$ & 283 & 31.5 & 2331 & 72.3 & 8 \\
\cline { 2 - 7 } & $\sigma, 10^{-3} \mathrm{Sm} / \mathrm{m}$ & 7.87 & 0.88 & 64.81 & 2.01 & 0.22 \\
\hline \multirow{3}{*}{$f=500 \mathrm{~Hz}$} & $\varepsilon^{\prime}, 10^{4}$ & 0.95 & 18.4 & 22.8 & 13.3 & 2.3 \\
\cline { 2 - 7 } & $\varepsilon^{\prime \prime}, 10^{4}$ & 23.8 & 13 & 243 & 12.3 & 2.4 \\
\cline { 2 - 7 } & $\sigma, 10^{-3} \mathrm{Sm} / \mathrm{m}$ & 8.66 & 3.6 & 71.2 & 3.4 & 0.7 \\
\hline
\end{tabular}


Based on the data (see Table 1) it can be concluded that there is a sharp increase in the absorption properties of the sample at a concentration $\sim 0.05 \%$ at lower frequencies. There is also strongly marked maximum in dispersion of electromagnetic waves for all concentrations over a range of $\sim 10 \mathrm{kHz}$. The behavior of $\varepsilon^{\prime}$ is associated with a decrease in the electromagnetic field change rate in comparison to the typical recharge time (relaxation time) of effective capacitor consisting of adjacent conductive particles. At higher frequencies, the capacitor does not have time to recharge, and the dielectric permittivity value tends to approach the same composite permittivity value with zero concentration of CNTs.

The dependence of the imaginary parts of permittivity and conductivity on the frequency also shows a clear concentrational nonlinearity. At $n=0.5 \%$, the transition into a zone of agglomerate conductivity occurs where there is deterioration in the conductivity of the sample. With an increase in the electromagnetic field frequency, the conductivity is caused by the bias current and increases for all samples. This nonlinear dependence suggests a restructuring of a complex fine powder $\mathrm{SiO}_{2}+$ multiwalled CNTs $+\mathrm{H}_{2} \mathrm{O}$ (free and bound) system. Structures are formed (agglomerates) at higher concentrations. The samples dielectric properties support this hypothesis. Their surface areas are less than the total surface area of their components. The reason for the formation of these structures is the large difference between silicon and CNTs surface energies.

Next, we sintered silica powder with CNT in an oven at high temperatures. The conductivity of samples was estimated by the resistance measurement of calibration samples with a cable tester. It is known [1,2], that CNTs themselves could often be quite good conductors.

We determined the concentration dependence of the conductivity $\sigma$. The results of these measurements are shown in Table 2.

TABLE 2. The dependence of conductivity upon CNT concentration (\%) in a nanocomposite with a $\mathrm{SiO}_{2}$ matrix

\begin{tabular}{|c|c|c|c|c|c|c|}
\hline $\mathrm{n}, \%$ & $\mathrm{REF}, 0$ & 0.05 & 0.5 & 1 & 3 & 5 \\
\hline$\sigma, 10^{-3} \cdot \mathrm{Sm} / \mathrm{m}$ & $<10^{-6}$ & $<10^{-6}$ & $<10^{-6}$ & 10 & 30 & 30 \\
\hline
\end{tabular}

Based on data acquired, a conclusion about percolation character of direct current conductivity in the obtained samples can be made. Up to a concentration of $0.1 \%$, there was no direct contact opening the conductivity channel. The conductivity significantly increased at concentrations above $0.1 \%$. REF is a ceramic sample without CNT. It's worth noting that the percolation threshold is clearly expressed only for direct current conductivity. Previously made alternating current measurements [2], though pointing at a concentration dependence, had no percolation character. This is explained by the absence of necessity for direct contact between CNTs at alternating current conductivity. The tubes are recharging, like the plates of a capacitor separated by an insulator.

\section{Positron Annihilation Spectroscopy studies}

Positron annihilation spectroscopy (PAS) is a successive method usually recommended for the detection of open volumes in materials. In order to verify the PAS potential application in further studies of our nanocomposites, measurements of the Doppler Broadening of Annihilation Line (DB) were performed. A variable-energy positron beam (VEP), available at the LEPTA facility [8] of the Joint Institute for Nuclear Research in Dubna, was used in this experiment. This is a small linear accelerator (linac) which uses frozen $\mathrm{Ne}$ as the moderator of positrons emitted from a ${ }^{22} \mathrm{Na}$ isotope. Then, it allows them to accelerate to the desired energies 
ranging from $50 \mathrm{eV}$ to $30 \mathrm{keV}$ and implant them into the sample in the form of a beam. The controllability of positron energies makes it possible to implant the particles at a precise depth. DB measurements, consisting of registration of energetic spectrum of gamma quanta emitted in annihilation processes were done using $\mathrm{HpGe}$ detector with energy resolution of $1.2 \mathrm{keV}$ at $511 \mathrm{keV}$. Analysis of obtained spectra results in extracting so called S parameter. This is defined as the ratio of area under the central part of $511 \mathrm{keV}$ line to the total area below this line. $S$ parameter represents a quantity related to the amount and size of free volume. More information about the method is presented e.g. here $[9,10]$.

In Fig. 2, the $S$ parameter dependence on energy for a reference sample without CNTs (circles) and a sample containing $1 \%$ CNTs (squares) are presented. It should be noted that differences between these dependencies are easily visible. The $S$ parameter values for the specimen containing CNTs are much lower. A similar trend was observed by Chen et. al. [11]. In this case, the decreasing $\mathrm{S}$ parameter was explained as the presence of additional carbon nanofiber particles which have no free volume. Further studies in this area are recommended.

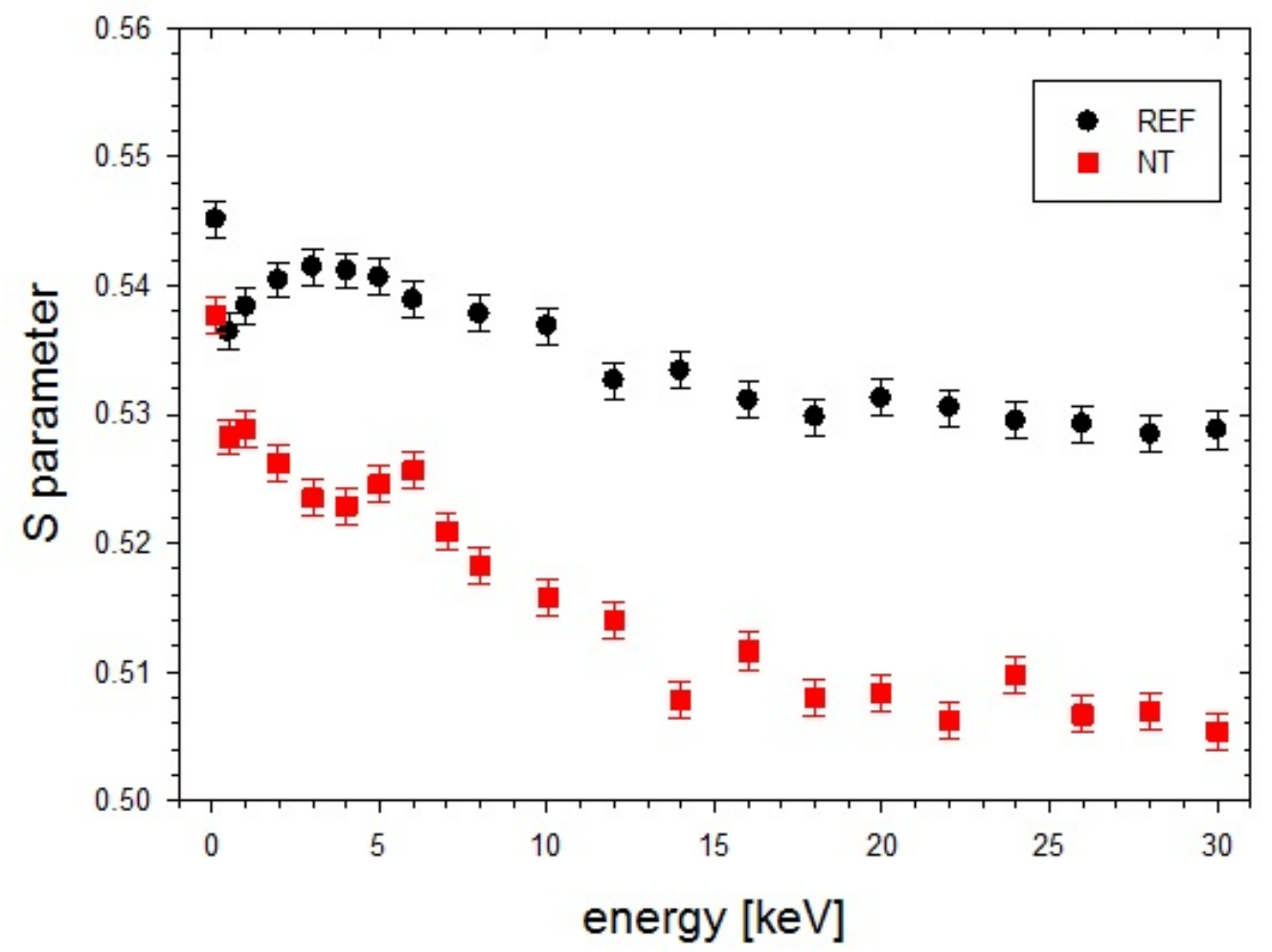

FIG. 2. The dependence of $S$-parameter of annihilation on positron energy for ceramic samples with CNTs concentration $1 \%-\mathbf{a}$. REF - sample without CNTs

- •. DALB spectra

\section{Conclusions}

All the given studies show that a nanocomposites properties are dependent upon the concentration (mass percentage) of CNTs. Although, during the investigation of the direct current conductivity, there was a percolation threshold located between 0.5 and $1 \%$. Other methods allowed the distinguishing of samples with lower CNTs concentrations. 


\section{Acknowledgements}

This work was supported by the project of the Ministry of Education of the Russian Federation No. 3635 "Investigation of the nanocomposites properties with controlled modification of the structure reinforced with carbon nanotubes".

\section{References}

[1] Eletskii A.V. Carbon nanotubes. Physiks Uspekhi, 1997, 40(9), P. 899-924.

[2] Eletskii A.V., Knizhnik A.A., Potapkin B.V., Kenny J.M. Electrical characteristics of carbon nanotube doped composites. Physiks Uspekhi, 2015, 58(3), P. 209-251.

[3] Rakov E.G. Carbon nanotubes in new materials. Russian Chem. Rev., 2013, 82(1), P. $27-47$.

[4] Muradyan V.E., Sokolov E.A., Babenko S.D., Moravsky A.P. Microwave dielectric properties of composites modified by carbon nanostructures. Techn. Phys., 2010, 55(2), P. 242-246.

[5] Usanov D.A., Skripal' A.V., Romanov A.V. Complex permittivity of composites based on dielectric matrices with carbon nanotubes. Techn. Phys., 2011, 56(1), P. 102-106.

[6] Usanov D.A., Skripal' A.V., Romanov A.V. Effect of annealing on the microwave characteristics of carbon nanotubes and the nanocomposite materials based on them. Techn. Phys., 2014, 59(6), P. 873-878.

[7] Kablov E.N., Kondrashov S.V., Yurkov G.Yu. Prospects of using carbonaceous nanoparticles in binders for polymer composites. Nanotechnologies in Russia, 2013 8(3-4), P. 163-185.

[8] Sidorin A.A., Meshkov I., Ahmanova E., Eseev M., Kobets A., Lokhmatov V., Pavlov V., Rudakov A., Yakovenko S. The LEPTA facility for fundamental studies of positronium physics and positron spectroscopy. Mater. Sci. Forum, 2013, 733, P. 291-296.

[9] Puska M.J., Nieminen R.M. Theory of positrons in solids and on solid surfaces. Rev. Mod. Phys., 1994, 66, P. 841-899.

[10] Krause-Rehberg R., Leipner S.H. Positron annihilation in semiconductors. Springer, Berlin, 1998.

[11] Chen H.M. Awad S., Jean Y.C., Yang J., James Lee L. Positron annihilation studies in polymer nanocomposites. Application of Accelerators in Research and Industry, 2011, 1336, P. 444-447. 